\title{
BOUNDED PROJECTIONS ON FOURIER-STIELTJES TRANSFORMS
}

\author{
CHARLES F. DUNKL AND DONALD E. RAMIREZ ${ }^{1}$
}

\begin{abstract}
We study certain algebraic projections on the measure algebra (of a locally compact abelian group) which extend to bounded projections on the uniform closure of the Fourier-Stieltjes transforms. These projections arise by studying a Raikov system of subsets induced by locally compact subgroups. These results generalize the inequality $\left\|\hat{\mu}_{d}\right\|_{\infty} \leqq\|\hat{\mu}\|_{\infty}$ (where $\mu$ is in the measure algebra, $\mu_{d}$ is the discrete part of $\mu$, and $\|\hat{\mu}\|_{\infty}$ is the sup-norm of the Fourier-Stieltjes transform).
\end{abstract}

Here $H$ will be a locally compact abelian (LCA) group. The group $H$ with the discrete topology is denoted $H_{d}$. This is the same as giving $H$ the topology induced from declaring the subgroup $G=\{0\} \subset H$ to be open. The space of finite regular Borel measures on $H$ is denoted $M(H)$. For $\mu \in M(H)$, let $\mu_{d}$ denote the discrete part of $\mu$. The ring homomorphism $\mu \mapsto \mu_{d}$ maps $M(H)$ onto $M\left(H_{d}\right)$, and this map is norm-nonincreasing in the measure norm; that is, $\left\|\mu_{d}\right\| \leqq\|\mu\|, \mu \in M(H)$. For $\mu \in M(H)$, we let $\hat{\mu}$ denote the Fourier-Stieltjes transform of $\mu$; that is

$$
\hat{\mu}(\gamma)=\int_{H}(\gamma(x))^{-} d \mu(x),
$$

$\gamma \in \hat{H}$ (the dual of $H$ ). In two previous papers [2], [3], we showed (in a more general setting) $\left\|\hat{\mu}_{d}\right\|_{\infty} \leqq\|\hat{\mu}\|_{\infty}, \mu \in M(H)$ (where $\|\cdot\|_{\infty}$ denotes the sup-norm). This further implies that $\mathcal{M}(\hat{H})=\mathcal{M}_{c}(\hat{H}) \oplus \mathcal{M}_{d}(\hat{H})$, where $\mathcal{H}(\hat{H}), \mathcal{M}_{c}(\hat{H})$, and $\mathcal{H}_{d}(\hat{H})$ are the sup-norm closures on $\hat{H}$ of the Fourier-Stieltjes transforms of measures from $M(H), M_{c}(H)$ (the space of continuous measures), and $M\left(H_{d}\right)$ respectively. Let $\Delta$ denote the maximal ideal space of $M(H)$, and let $\kappa \hat{H}$ denote the $\Delta$-closure of $\hat{H}$ in $\Delta$. (Recall $\hat{H} \subset \Delta$ under the identification map from $\hat{H}$ to $\Delta$ by $\pi_{\gamma}(\mu)=\hat{\mu}(\gamma)$, $\gamma \in \hat{H}, \mu \in M(H)$.) We call the set $\kappa \hat{H} \backslash \hat{H}$ the fringe of $\hat{H}$. The result

Received by the editors February 8, 1971 .

AMS 1970 subject classifications. Primary 43A10, 43A25.

Key words and phrases. Measure algebra of a locally compact abelian group, FourierStieltjes transform, Raikov system of subsets, positive definite function.

${ }^{1}$ This research was supported in part by NSF contract number GP-19852. 
$\left\|\hat{\mu}_{d}\right\|_{\infty} \leqq\|\hat{\mu}\|_{\infty}(\mu \in M(H))$ implies that the fringe of $\hat{H}$ contains a homeomorphic copy of the Bohr group $\beta \hat{H}$ of $\hat{H}$ (under the map $\chi \mapsto \pi_{\chi}$ from $\beta \hat{H}$ to $\Delta$ given by $\left.\pi_{\chi}(\mu)=\int_{H} \bar{\chi} d \mu_{d}, \mu \in M(G), \chi \in \beta \hat{H}\right)$.

The setting in this paper is as follows. We let $H$ be an LCA group with topology $\mathfrak{C}_{H}$, and $G$ a subgroup of $H$ which has an LCA group topology $\mathfrak{C}_{G}$ such that the injection $\left(G, \mathfrak{C}_{G}\right) \rightarrow\left(H, \mathfrak{C}_{H}\right)$ is continuous. For example, suppose $G$ is the image under a continuous monomorphism of an LCA group. We let $H_{G}$ denote $H$ with the topology induced by declaring the subgroup $G$ with the $\mathfrak{C}_{G}$-topology to be open. We will assume that $G$ is a nonopen subgroup of $H$ so that $H \neq H_{G}$ topologically.

We now will define the natural projection $P: M(H) \rightarrow M\left(H_{G}\right)$ by utilizing a Raikov system of subsets of $H$. (For the basic facts concerning Raikov systems see [5].) Let $\mathscr{F}$ denote a family of $\sigma$-compact subsets of $H$ such that: (1) if $A \in \mathscr{F}, B$ is $\sigma$-compact, and $B \subset A$, then $B \in \mathcal{F}$, (2) if $\left\{A_{n}\right\}_{n=1}^{\infty} \subset \mathcal{F}$, then $\bigcup_{n=1}^{\infty} A_{n} \in \mathscr{F},(3)$ if $A, B \in \mathscr{F}$, then $A+B \in \mathcal{F}$, and (4) if $A \in \mathscr{F}$ and $x \in H$, then $x+A \in \mathscr{F}$. Such a family of subsets of $H$ is called a Raikov system. We choose $\mathcal{F}$ to be the Raikov system generated by the family of compact subsets of $G$.

Let $R$ be the set of measures $\mu \in M(H)$ such that $|\mu|$ is concentrated on some elements of $\mathcal{F}$, and let $I$ be the set of measures $\mu \in M(H)$ such that $|\mu|(A)=0$ for all $A \in \mathscr{F}$. Then $I$ is a closed ideal in $M(H)$ and $R$ is a closed subalgebra of $M(H)$. Furthermore, $M(H)=R \oplus I$ (see, for example, [5, p. 151]). Now $R$ can be identified with $M\left(H_{G}\right)$, and thus the natural projection $P: M(H) \rightarrow M\left(H_{G}\right)$ is induced by the given direct sum. For $\mu \in M(H)$, we write $\mu=\mu_{G}+\mu_{I}$ where $\mu_{G} \in M\left(H_{G}\right)$ and $\mu_{I} \in I$. Thus $P \mu=\mu_{G}, \mu \in M(H)$. Observe that $P$ is a norm-bounded projection; that is, $\|P \mu\| \leqq\|\mu\|, \mu \in M(H)$. Our goal now is to show

$$
\left\|(P \mu)^{\wedge}\right\|_{\infty} \leqq\|\hat{\mu}\|_{\infty}, \quad \mu \in M(H) .
$$

Let $\phi: H_{G} \rightarrow H$ be the identity map and $\hat{\phi}: \hat{H} \rightarrow \hat{H}_{G}$ the adjoint map (an injection). In an earlier paper [4], we showed for any continuous homomorphism $\pi: G_{1} \rightarrow G_{2}\left(G_{1}, G_{2}\right.$ LCA groups) that $\pi$ is open if and only if $\hat{\pi}: \hat{G}_{2} \rightarrow \hat{G}_{1}$ (the adjoint map) is proper (the inverse image of a compact set is compact). Thus since $\phi$ is not open, $\hat{\phi}$ is not proper. The map $\phi$ induces a continuous homomorphism $\phi^{*}: M\left(H_{G}\right) \rightarrow M(H)$. Since $\phi$ is one-to-one, $\hat{\phi} \hat{H}$ is dense in $\hat{H}_{G}$. Indeed for any compact $K \subset \hat{H}$, $\hat{\phi}(\hat{H} \backslash K)$ is dense in $\hat{H}_{G}$. For $\mu \in M\left(H_{G}\right),\|\hat{\mu}\|_{\infty}$ is the supremum of $|\hat{\mu}|$ over either $\hat{\phi} \hat{H}$ or $\hat{H}_{G}$. (We will identify $\hat{\phi} \hat{H}$ and $\hat{H}$ as subsets of $\hat{H}_{G}$ when convenient.)

For an LCA group $L$, we let $P(L)$ denote the space of continuous positive definite functions on $L$; we let $P_{c}(L)$ be those $f \in P(L)$ with compact support. 
We will denote the Haar measure on $H_{G}$ by $\lambda$. (The measure $\lambda$ restricted to $G$ is the Haar measure on $G$.)

Proposition 1. Let $f \in P_{c}\left(H_{G}\right)$ and let $d \mu=f d \lambda$. If $g \in P_{c}(H)$, then $g * \mu$ (convolution in $M(H)$ ) is in $P_{c}(H)$.

Proof. Since $f \in P_{c}\left(H_{G}\right), \hat{f} \in L^{1}\left(\hat{H}_{G}\right)$ by the inversion theorem [7, p. 22], and $\hat{f} \geqq 0$ by Bochner's theorem [7, p. 19]. Thus for $\gamma \in \hat{H} \subset \hat{H}_{G}$, $\hat{\mu}(\gamma)=\int_{H} \bar{\gamma} d \mu=\int_{H_{G}} \bar{\gamma} f d \lambda=\hat{f}(\gamma) \geqq 0$.

Since $g$ and $\mu$ have compact supports, $g * \mu$ is a continuous function on $H$ with compact support. Finally, $g * \mu$ is positive definite since $(g * \mu)^{\wedge}=$ $\hat{g} \hat{\mu} \geqq 0$ on $\hat{H}$.

An LCA group $L$ is amenable, and thus satisfies the condition of Godement: the constant function 1 can be approximated uniformly on compact subsets of $L$ by functions of the form $k * \tilde{k}$, where $k$ is a continuous function with compact support and $\tilde{k}(x)=(k(-x))^{-}, x \in L$. (See $[6$, p. 168, 172].) Thus we have:

Proposition 2. Let $L$ be an LCA group and $K \subset L$ a compact subset of L. Given $\varepsilon>0$, there is $p \in P_{c}(L)$ such that $p(0)=1$ and $|p-1|<\varepsilon$ on $K$.

Proposition 3. Let $K$ be a compact subset of $H_{G}$, and let $U$ be $a$ relatively compact neighborhood of 0 in $H_{G}$. Then there is a neighborhood $V$ of 0 in $H$ such that $(x+V) \cap K \subset x+U$ for all $x \in K$.

Proof. Since $K$ is compact in $H_{G}, K-K$ is also compact in $H_{G}$; and the induced topology on $K-K$ from $H$ agrees with the $H_{G}$-topology on $K-K$ (since compact topologies are minimal Hausdorff). Thus there is an $H$-open neighborhood of $0, V$, such that $V \cap(K-K) \subset$ $U \cap(K-K)$. Thus for $x \in K,(x+V) \cap K \subset x+(V \cap(K-\{x\})) \subset$ $x+(V \cap(K-K)) \subset x+(U \cap(K-K)) \subset x+U$.

Proposition 4. Let $\xi \in \hat{H}_{G}, K$ a compact subset of $H_{G}$, and $\varepsilon>0$ be given. Then there exists $\gamma \in \hat{H}$ such that $|\gamma-\xi|<\varepsilon$ on $K$.

Proof. Recall that $\hat{\phi} \hat{H}$ can be identified with $\hat{H}$, and it is dense in $\hat{H}_{G}$. Finally, the topology in $\hat{H}_{G}$ is the compact-open topology.

TheOREM 5. Let $P: M(H) \rightarrow M\left(H_{G}\right)$. Then $\left\|(P \mu)^{\wedge}\right\|_{\infty} \leqq\|\hat{\mu}\|_{\infty}$, $\mu \in M(H)$.

Proof. Let $\mu \neq 0$ be in $M(H)$, and let $\xi \in \hat{H}_{G}$. Write $\mu=\mu_{G}+\mu_{I}$ where $\mu_{G} \in M\left(H_{G}\right)$ and $\mu_{I} \in I$ using the previously described Raikov system. We will show $\left|\hat{\mu}_{G}(\xi)\right| \leqq\|\hat{\mu}\|_{\infty}$. 
We may assume spt $\mu_{G}$ (spt denotes the support) is compact in $H_{G}$. By Proposition 2, there is $p \in P_{c}\left(H_{G}\right)$ such that $p(0)=1$ and $|p-1|<$ $\varepsilon /\|\mu\|$ on spt $\mu_{G}$.

Since $\left|\mu_{I}\right|(\operatorname{spt} p)=0$, we may assume spt $\mu_{I} \cap \operatorname{spt} p=\varnothing$. Since $p$ is uniformly continuous in the $H_{G}$-topology, there is a $H_{G}$-open neighborhood of $0, U$, such that for $x \in H_{G}$ and $y \in U,|p(x+y)-p(x)|<$ $\varepsilon /\|\mu\|$. Let $K=-K$ be a compact subset of $H_{G}$ containing spt $p$ and spt $\mu_{G}$. By Proposition 3, choose $V$ to be an $H$-open neighborhood of 0 such that $V=-V$ and $(x+V) \cap K \subset x+U$ for all $x \in K$; we further assume that (spt $p+V) \cap\left(\right.$ spt $\left.\mu_{I}+V\right)=\varnothing$.

Now choose $\gamma \in \hat{H}$ by Proposition 4 such that $|\gamma-\bar{\xi}|<\varepsilon /\|\mu\|$ on $K$; and choose $g \in P_{c}(H)$ with spt $g \subset V, g \geqq 0$, and $\int_{U} g d \lambda=1$. For any $x \in K, \quad|(g * p d \lambda)(x)-p(x)|=\left|\int_{V} g(y) p(x-y) d \lambda(y)-p(x)\right|=$ $\left|\int_{U} g(y)(p(x-y)-p(x)) d \lambda(y)\right|<\varepsilon /\|\mu\|$ (since $V \cap(x-K) \subset U, x \in$ spt $p$ ). Thus letting $f=g * p d \lambda$, spt $f \subset V+\operatorname{spt} p$ and $f \in P_{c}(H)$ (by Proposition 1). Also $f(0)<p(0)+\varepsilon /\|\mu\|=1+\varepsilon /\|\mu\|$, and spt $f \cap$ spt $\mu_{I}=\varnothing$. For $x \in \operatorname{spt} \mu_{G}$,

And

$$
|f(x)-1| \leqq|f(x)-p(x)|+|p(x)-1|<2 \varepsilon /\|\mu\| .
$$

$$
\begin{aligned}
\left|\int_{H_{G}} \bar{\xi} d \mu_{G}-\int_{H} \gamma f d \mu\right| \leqq & \left|\int_{H_{G}} \bar{\xi} d \mu_{G}-\int_{H_{G}} \gamma d \mu_{G}\right| \\
& +\left|\int_{H_{G}} \gamma d \mu_{G}-\int_{H} \gamma f d \mu_{G}\right|+\left|\int_{H} \gamma f d \mu_{T}\right| \\
& <(\varepsilon /\|\mu\|)\left\|\mu_{G}\right\|+(2 \varepsilon /\|\mu\|)\left\|\mu_{G}\right\|+0 \\
& \leqq 3 \varepsilon .
\end{aligned}
$$

Now $\left|\int_{H} \gamma f d \mu\right| \leqq f(0)\|\hat{\mu}\|_{\infty}<(1+\varepsilon /\|\mu\|)\|\hat{\mu}\|_{\infty}$ (since $\gamma f$ is positive definite).

Summarizing, given $\xi \in \hat{H}_{G}$,

$$
\begin{aligned}
\left|\hat{\mu}_{G}(\xi)\right| & =\left|\int_{H_{G}} \bar{\xi} d \mu_{G}\right| \leqq\left|\int_{H} \gamma f d \mu\right|+3 \varepsilon \\
& \leqq(1+\varepsilon /\|\mu\|)\|\hat{\mu}\|_{\infty}+3 \varepsilon \leqq\|\hat{\mu}\|_{\infty}+4 \varepsilon .
\end{aligned}
$$

And so $\left\|\hat{\mu}_{G}\right\|_{\infty} \leqq\|\hat{\mu}\|_{\infty}$.

Corollary 6. Let $\mathcal{H}(\hat{H}), \mathcal{M}\left(\hat{H}_{G}\right)$ and J denote the uniform closures of the Fourier-Stieltjes transforms of $M(H), M\left(H_{G}\right)$, and I respectively. Then $\mathcal{M}(\hat{H})=\mathcal{M}\left(\hat{H}_{G}\right) \oplus J$.

Corollary 7. If $\mu \in M(H)$ and $\hat{\mu} \in \mathcal{M}\left(\hat{H}_{G}\right)$, then $\mu \in M\left(H_{G}\right)$. 
Corollary 8. Let $\hat{H}_{G}$ be embedded in $\kappa \hat{H}$ (the maximal ideal space of $\mathcal{H}(\hat{H})$; equivalently, the closure of $\hat{H}$ in $\Delta)$, by $\gamma \mapsto \pi_{\gamma}$ from $\hat{H}_{G}$ to $\kappa \hat{H}$ where $\pi_{\gamma}(\mu)=\hat{\mu}_{G}(\gamma)(\mu \in M(H))$. Since $\pi_{\gamma}(\mu)=0$ for $\mu \in L^{\mathbf{1}}(H)$ (recall $G$ is nonopen in $H$ ), $\pi_{\gamma} \in \kappa \hat{H} \backslash \hat{H}$ (the fringe of $\hat{H}$ ). In particular, for $\mu \in M(H),\left\|\hat{\mu}_{G}\right\|_{\infty}=\lim \sup \left\|\hat{\mu}_{G}\right\|_{\infty} \leqq\|\hat{\mu}\|_{\infty}$.

These corollaries follow from the inequality $\left\|\hat{\mu}_{G}\right\|_{\infty} \leqq\|\hat{\mu}\|(\mu \in M(H))$. The proofs are discussed in a more general setting in [3]. For $G=\{0\}$ (and thus $H_{G}=H_{d}$ ), Corollary 7 is due to E. Hewitt for $H$ with a restricted hypothesis and to W. Eberlein in general. A reference for these facts, plus a different (although closely related) direct sum decomposition, is [1].

Some interesting examples of LCA groups $H$ with a nonopen subgroup $G$ are: (1) $H$ nondiscrete and $G=\{0\}$, (2) $G$ noncompact and $H=\beta G$ the Bohr compactification of $G$, (3) $G=R$ (the real numbers) and $H$ a compact solenoidal group, and (4) certain local direct product groups embedded in the appropriate complete direct product groups.

\section{BIBLIOGRAPHY}

1. R. Burckel, Weakly almost periodic functions on semigroups, Gordon and Breach, New York, 1970.

2. C. Dunkl and D. Ramirez, $C^{*}$-algebras generated by measures, Bull. Amer. Math. Soc. 77 (1971), 411-412.

3.,$- C^{*}$-algebras generated by Fourier-Stieltjes transforms, Trans. Amer. Math. Soc. (to appear).

4. - Homomorphisms on groups and induced maps on certain algebras of measures, Trans. Amer. Math. Soc. 160 (1971), 475-485.

5. E. Hewitt, The asymmetry of certain algebras of Fourier-Stieltjes transforms, Michigan Math. J. 5 (1958), 149-158. MR 21 \#4993.

6. H. Reiter, Classical harmonic analysis and locally compact groups, Oxford Math. Monographs, Clarendon Press, Oxford, 1968.

7. W. Rudin, Fourier analysis on groups, Interscience Tracts in Pure and Appl. Math., no. 12, Interscience, New York, 1962. MR 27 \#2808.

Department of Mathematics, University of Virginia, Charlottesville, VIRGINIA 22903 